\title{
Quantum and classical dynamics of a diatomic molecule in laser fields with frequency in the region producing maximum dissociation
}

\author{
K. I. Dimitriou, ${ }_{1}^{1,2}$ V. Constantoudis, ${ }^{3}$ Th. Mercouris, ${ }^{2}$ Y. Komninos, ${ }^{2}$ and C. A. Nicolaides ${ }^{1,2}$ \\ ${ }^{1}$ Physics Department, National Technical University, Athens, Greece \\ ${ }^{2}$ Theoretical and Physical Chemistry Institute, National Hellenic Research Foundation, \\ 48 Vasileos Constantinou Avenue, Athens 11635, Greece \\ ${ }^{3}$ Institute of Microelectronics, NCSR “Demokritos," Aghia Paraskevi 15310, Greece
}

(Received 4 June 2007; published 17 September 2007)

\begin{abstract}
By solving nonperturbatively the equations of Schrödinger and of Hamilton, we have studied the timedependent multiphoton dissociation of a diatomic molecule induced by a moderate low-frequency laser field. The photodissociation probabilities are calculated and analyzed as a function of the laser frequency, the intensity, and the pulse shape. A well-established quantum and classical result is that for laser intensities of the order of $10^{14} \mathrm{~W} / \mathrm{cm}^{2}$ the dissociation probability presents a maximum at the frequency $\omega_{\max }$ $\sim(0.80-0.90) \omega_{01}$, where $\omega_{01}$ is the transition frequency from the ground to the first excited vibrational state (redshift phenomenon). In this work, we go further and explore the quantum and classical effects of radiation at the optimum frequency $\omega_{\max }$ on the overall vibrational excitation and dissociation dynamics. First, it is shown that both quantum and classical results predict that $\omega_{\max }$ continues to be the optimum frequency for photodissociation even at the order of $10 \mathrm{TW} / \mathrm{cm}^{2}$, i.e., low intensities. The quantum results show a multipeak structure versus laser frequency, which is attributed to resonant multiphoton transitions, while the classical results show a smooth curve with a broad maximum at $\omega_{\max }$ which is explained by phase space arguments. Second, it is found that in both quantum and classical approaches $\omega_{\max }$ marks a transition in the effects of turn-on time of the pulse shape on dissociation probability: for $\omega<\omega_{\max }$ the gradual turn-on of the field leads to a noticeable reduction of the photodissociation probability, while for $\omega>\omega_{\max }$ this effect is of minor importance. A classical interpretation of this finding is given, which is based on stickiness effects in phase space. Finally, the crucial role of $\omega_{\max }$ is further demonstrated in the time dependence of bound-state occupation probabilities. The total survival probability decreases faster with time for $\omega<\omega_{\max }$ rather than for $\omega$ $>\omega_{\max }$. Further, for $\omega>\omega_{\max }$ the bound-state occupation probabilities exhibit multiphoton Rabi-type oscillations where more than two vibrational states are involved. These phenomena are predicted by both quantum and classical dynamics, although there are secondary differences which are revealed and discussed.
\end{abstract}

DOI: $10.1103 /$ PhysRevA.76.033406

PACS number(s): 33.80.Wz, 33.80.Gj, 05.45.Mt, 33.80.Rv

\section{INTRODUCTION}

A well-known issue in physics is understanding theoretically and quantitatively the various aspects of the correspondence between quantum and classical mechanics. The validity of this type of understanding is enhanced when real physical systems rather than simple models-albeit mathematically convenient-are the object of investigation. For example, one such system whose analysis has produced useful and pioneering results in the area of chaotic dynamics is the microwave ionization of highly excited hydrogen atoms [1-6].

The present contribution is in this spirit, the purpose of the investigation having been to treat the problem of laserinduced time-dependent multiphoton dissociation of the diatomic Morse molecule by advanced methods and analysis of both classical $(\mathrm{CM})$ and quantum mechanics $(\mathrm{QM})$. The results and analysis that are reported here constitute a continuation of our earlier investigations on this subject, which focused on aspects of classical nonlinear and chaotic dynamics [7-9]. Now, in addition to further results and analysis from $\mathrm{CM}$, we have obtained additional quantitative information by solving nonperturbatively the time-dependent Schrödinger equation (TDSE) for the description of the photodissociation of a Morse molecule, (with parameters for the
HF molecule), in a low-frequency laser field. Given the two sets of results, it is possible to have general pictures for aspects of classical-quantum correspondence for the dynamics of a physical system with a discrete and continuous spectrum.

Previous studies concerning the quantum dynamics of the Morse diatomic molecule driven by laser pulse were based on the numerical solution of the TDSE on a grid [10-14]. In this method, the wave function is discretized in the position space and its time evolution is calculated according to the split-operator method [10]. In the present work, we implemented the state-specific expansion approach (SSEA), a wave-function-based method developed for atoms and small molecules $[15,16]$. The basis of this approach relies on the fundamental principle of quantum mechanics that the timedependent state of the atomic or molecular system is written as a linear combination of the eigenstates of the discrete and continuous unperturbed atomic or molecular spectrum with time-dependent coefficients. This approach has the advantage that at each time point the occupation probability of each state is known directly. Furthermore, when the scattering states of the continuous spectrum are energy normalized, the numerical and interpretative difficulties characterizing methods where the whole system is enclosed in an artificial box and the states are obtained by diagonalization of the 
Hamiltonian on a large basis set, are avoided. We note that an expansion approach with explicit consideration of the scattering wave function was recently also used by Lima and Hornos [17] for the study of the multiphoton dissociation of $\mathrm{OH}$.

The application presented here involved the nonperturbative computation from first principles of the time-dependent photodissociation probability of the HF molecule, for the frequency region where photodissociation is maximized, as a function of field intensity and pulse shape. According to both quantum and classical predictions, this region is about $\omega_{\max }$ $\simeq 0.86 \omega_{01}[7,8,12,13,16,18]$. We show that the quantum photodissociation probability as a function of laser frequency exhibits a multipeak structure, especially at high intensities, which is attributed to resonance transitions. The classical one is found to be a smooth curve with a maximum located near the maximum quantum peak, and it is explained by phase space arguments (Sec. III A). We also address the role that the pulse shape plays on the photodissociation probability. Goggin and Milonni [19] found that both CM and QM predict a reduction of the excitation energy when a gradual turn-on of the field is used, compared to that which is obtained assuming an ac field. In this study, we show that in both CM and QM the effect of the pulse shape on the photodissociation probability depends on the laser frequency. In particular, for $\omega<\omega_{\max }$ the use of a gradual turn-on of the field reduces significantly the photodissociation probability, while for $\omega>\omega_{\max }$ this effect is of minor importance (Sec. III B). Finally, we examine the time dependence of the survival and the bound-state occupation probabilities as a function of laser frequency (Sec. III C). Again, in both CM and QM the role of $\omega_{\max }$ is crucial: for $\omega<\omega_{\max }$ the total survival probability decreases faster with time than for $\omega>\omega_{\max }$. Moreover, for $\omega>\omega_{\max }$ the bound-state occupation probabilities exhibit multiphoton Rabi-type oscillations where more than two vibrational states are involved.

\section{DESCRIPTION OF THE SYSTEM AND METHODOLOGY}

We will study the multiphoton dissociation of a diatomic molecule in one dimension, where only the vibrational motion is considered. This motion is described by the Morse potential [20]

$$
V_{M}(x)=D\left\{1-\exp \left[-\alpha\left(x-x_{0}\right)\right]\right\}^{2}
$$

where $D=0.2101$ a.u. is the dissociation energy, $\alpha=1.22$ is the range of the molecular potential, and $x_{0}=1.75$ is the equilibrium distance. The values of these parameters are taken from $[13,21]$ and correspond to the ground electronic state of the HF molecule. This system contains 22 bound vibrational states. In other molecules for which multiphoton dissociation was studied previously $[11,16,22]$, the number of vibrational states was six [11], eight [16], and 179 [22].

In the semiclassical approximation, the Hamiltonian of a Morse molecule in the presence of a laser field in the electric dipole length gauge has the form

$$
H=\frac{p^{2}}{2 m}+V_{M}(x)+g(x) F(t),
$$

where $m$ is the reduced mass, $\omega$ is the laser frequency, $g(x)$ $=e\left(x-x_{0}\right)$ is the dipole moment, and $F(t)$ is the ac laser field,

$$
F(t)=F_{0} \sin (\omega t)
$$

with $F_{0}$ the field strength.

In this work, the dipole moment is considered in the linear approximation in an effort to complement, for a wide frequency region, the knowledge of the quantum-classical correspondence of the photodissociation of a Morse molecule, obtained from previous works $[19,23]$. This form allows the use of analytical formulas for the matrix elements of the bound-bound [23-27] and bound-continuum transitions $[23,26,27]$. On the other hand, the calculation of continuumcontinuum matrix elements in the length form presents difficulties due to singularities. In order to solve this problem we developed a methodology that is based on a combination of analytical and numerical methods.

In the next two sections we will describe the quantum and classical formalisms that were employed for the quantitative solution of the problem described by the Hamiltonian of Eq. (2).

\section{A. Quantum dynamics}

\section{Time evolution}

Our aim is to solve the one-dimensional TDSE

$$
i \hbar \frac{d \Psi(x, t)}{d t}=\hat{H}(t) \Psi(x, t)
$$

where $\hat{H}(t)$ is the Hamiltonian operator $(2)$, and $\Psi(x, t)$ is the wave function of the system. According to the SSEA [15], the wave function is written as a superposition of bound $\left[\Psi_{\nu}(x)\right]$ and energy-normalized continuum eigenstates $\left[\Psi_{E}(x)\right]$ of the molecule:

$$
\Psi(x, t)=\sum_{\nu=0}^{\nu_{\max }-1} a_{\nu}(t) \Psi_{\nu}(x)+\int b(E, t) \Psi_{E}(x) d E .
$$

Here, $\nu_{\max }=22$ corresponds to the number of vibrational states of the HF molecule. Using Eq. (5) the TDSE is transformed into a system of coupled integro-differential equations:

$$
\begin{aligned}
i \hbar \frac{d a_{\nu}(t)}{d t}= & \epsilon_{\nu} a_{\nu}(t)+F(t) \sum_{\mu} B(\nu, \mu) a_{\mu}(t) \\
& +F(t) \int b(E, t) D(\nu, E) d E, \\
i \hbar \frac{d b_{E}(t)}{d t}= & E b_{E}(t)+F(t) \sum_{\mu} D(E, \mu) a_{\mu}(t) \\
& +F(t) \int b\left(E^{\prime}, t\right) C\left(E, E^{\prime}\right) d E^{\prime},
\end{aligned}
$$


where $B(\nu, \mu), D(\nu, E)$, and $C\left(E, E^{\prime}\right)$ are the matrix elements for the bound-bound, bound-continuum, and continuumcontinuum electric dipole transitions, respectively. The set of equations (6) and (7) is written in a matrix form and is numerically calculated by expanding both the time-dependent coefficients and the laser field in Taylor series [15]. The integrals in energy are calculated using the standard technique of the trapezoidal rule, in conjunction with very small energy steps.

The photodissociation probability at a given time is defined as

$$
P_{D}^{q}(t)=1-\sum_{\nu=0}^{\nu_{\max }-1}\left|a_{\nu}(t)\right|^{2}
$$

where the summation is performed only on the bound-state coefficients.

\section{Matrix elements}

The dipole matrix elements for the bound-bound transitions $B(\nu, \mu)=\left\langle\nu\left|x-x_{0}\right| \mu\right\rangle$ are documented in the bibliography [23-27]. The off-diagonal diagonal matrix elements are

$$
B(\nu, \mu)=\frac{1}{\alpha} 2(-1)^{\mu-\nu+1} \frac{\left(\beta_{\nu} \beta_{\mu}\right)^{1 / 2}}{\beta_{\nu}^{2}-\beta_{\mu}^{2}}\left(\frac{\mu ! \Gamma\left(2 \beta_{\mu}+\mu+1\right)}{\nu ! \Gamma\left(2 \beta_{\nu}+\nu+1\right)}\right)^{1 / 2}
$$

where $\mu>\nu$. The diagonal matrix elements are given by

$$
\begin{aligned}
B(\nu, \nu)= & \frac{1}{\alpha}[\ln (2 \beta+2 \nu+1)+\psi(2 \beta+\nu+1) \\
& -\psi(2 \beta+1)-\psi(2 \beta)],
\end{aligned}
$$

where $\psi$ is the derivative of the logarithm of the $\Gamma$ function [28]. In the equations (9) and (10) the parameter is given by $\beta_{\nu, \mu}=\sqrt{2 m\left(-E_{\nu, \mu}\right)} / \alpha \hbar$, where $E_{\nu, \mu}$ is the energy of the $\nu$ th and $\mu$ th bound states respectively.

The matrix elements between bound and continuum states $D(\nu, E)=\left\langle\nu\left|x-x_{0}\right| E\right\rangle$ are also given by an analytical formula $[23,26,27]$,

$$
\begin{aligned}
D(\nu, E)= & \frac{1}{\sqrt{d E / d \epsilon}} \alpha \frac{(-1)^{\nu+1}\left|\Gamma\left(\frac{1}{2}-d+i \epsilon\right)\right|}{\pi\left(\beta^{2}+\epsilon^{2}\right)} \\
& \times\left(\frac{2 \beta \epsilon \sinh (2 \pi \epsilon)}{\nu ! \Gamma(2 \beta+\nu+1)}\right)^{1 / 2}|\Gamma(\beta+i \epsilon+\nu+1)|^{2}
\end{aligned}
$$

with $\epsilon=\sqrt{2 m E / \alpha^{2} \hbar^{2}}$.

The continuum-continuum matrix elements contain an onshell singularity of the form $1 /\left(E-E^{\prime}\right)^{2}$, when the two energies of the continuous spectrum, $E$ and $E^{\prime}$, become equal. This singularity arises from the contribution at large intermolecular distances, where the asymptotic form of the continuum wave functions of the free Morse molecule is that of a plane wave [26]. As we are interested in the dissociation process, these matrix elements play a crucial role in the calculation of the physical quantities. Following the analysis of
[15] for atomic potentials, in this work this singularity was treated systematically, in a computation that is carried out numerically (see the Appendix). We mention that, recently, an analytical expression was derived by Lima and Hornos [27], containing ${ }_{3} F_{2}$ functions. The complexity of such expressions suggests that, for economical computation, the numerical techniques that are used in the context of the SSEA are preferable.

\section{B. Classical dynamics}

The classical dynamics of the system is obtained by numerically integrating Hamilton's equations of motion:

$$
\dot{x}=\frac{p}{m},
$$

$$
\dot{p}=2 D \alpha \exp \left[-\alpha\left(x-x_{0}\right)\right)\left\{1-\exp \left(-\alpha\left(x-x_{0}\right)\right)\right\}+F(t) .
$$

For the generation of the initial position and momentum, we first perform a canonical transformation to the action-angle variables

$$
J=-\sqrt{\frac{2 \mu D}{\alpha^{2}}}(1-\sqrt{1-E}),
$$

$$
\theta=-\operatorname{sgn}(p) \arccos \left(\frac{1-E}{\sqrt{E}} \exp \left[-\alpha\left(x-x_{0}\right)\right]-\frac{1}{\sqrt{E}}\right),
$$

where $E=H_{0} / D<1$ is the dimensionless energy of the unperturbed molecule for the bound part, with $H_{0}=p^{2} / 2 m$ $+V_{M}(x)$, and $\operatorname{sgn}(p)=1$ for $p \geqslant 0, \operatorname{sgn}(p)=-1$ for $p<0$.

The initial ensemble of the classical trajectories is chosen according to the microcanonical distribution, where $E$ is constant and equal to the energy of the initial quantum vibrational state and $\theta$ is uniformly distributed in the domain $[-\pi, \pi][8]$. The stroboscopic plots of phase space in both $(x, p)$ and $(E, \theta)$ representations provide important information about the time evolution of the classical initial state. In the present work, they constitute the basic tools for the timedependent analysis of the classical dissociation process and its dependence on laser parameters.

During the time evolution of the interaction, dissociation occurs for those trajectories $N_{\text {diss }}$ with compensated energy $[2,19]$

$$
E_{c}=\frac{\left[p-\left(F_{0} / \omega\right) \cos (\omega t)\right]^{2}}{2 m}+V_{M}(x)>0 .
$$

The classical photodissociation probability at time $t$ is equal to

$$
P_{D}^{c}(t)=\frac{N_{\text {diss }}}{N_{t o t}},
$$

where $N_{\text {tot }}$ is the total number of trajectories. 


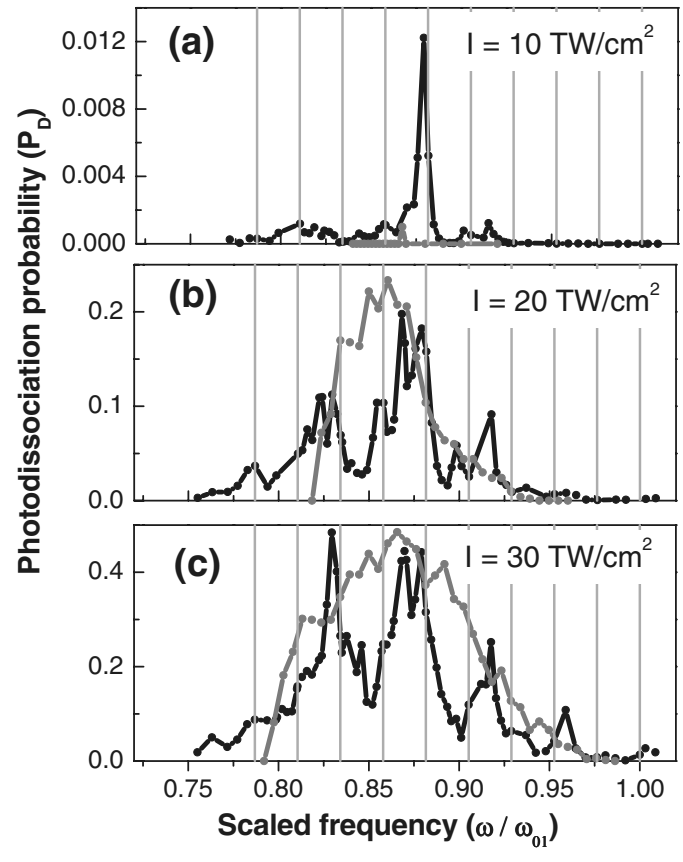

FIG. 1. Quantum (black line with full circles) and classical (gray line with full circles) photodissociation probability of HF as a function of the scaled laser frequency $\left(\omega / \omega_{01}\right)$ for three different laser intensities $I=(\mathrm{a}) 10$, (b) 20 , and (c) $30 \mathrm{TW} / \mathrm{cm}^{2}$. The vertical lines correspond to the scaled frequencies $\omega / \omega_{0 \nu}$ with $\omega_{0 \nu}=\left(E_{\nu}-E_{0}\right) / \nu$ and $\nu=0,1,2, \ldots, 10$ (see Fig. 3). The total time of interaction is $T_{a c}=13140$ a.u. and corresponds to 38 cycles for a laser frequency equal to the resonance frequency of $\omega_{01}$.

\section{RESULTS}

\section{A. Dependence on the laser intensity}

We have calculated quantal and classical photodissociation probabilities as a function of the scaled laser frequency $P_{D}\left(\omega / \omega_{01}\right)$ for the HF molecule interacting with an ac laser field [see Eq. (3)]. Results for laser intensities $I=10,20$, and $30 \mathrm{TW} / \mathrm{cm}^{2}$ are shown in Figs. 1(a)-1(c). Both quantal and classical results show a maximum of the photodissociation probability at laser frequencies that are redshifted with respect to the $\omega_{01}$ resonant frequency, having values in the range $(0.80-0.90) \omega_{01}$. A similar finding has been reported in [13], where a comparison of classical and quantal dissociation probabilities versus interaction time was also made. Further, in [8] a classical interpretation was provided in phase space terms. However, both studies were limited to strong laser fields, where, anyway, the quantum-classical correspondence is expected to manifest more distinctly [19]. Here, we explore the quantal and classical $P_{D}\left(\omega / \omega_{01}\right)$ for a range of laser intensities considerably lower than those of $[8,13]$. For the smallest intensity $I=10 \mathrm{TW} / \mathrm{cm}^{2}$, the quantum distribution presents one main peak located at $\omega / \omega_{01} \simeq 0.88$ and several side peaks of much lower magnitude, while the classical distribution exhibits a weaker spike around $\omega / \omega_{01} \simeq 0.86$ [see Fig. 1(a)]. Thus, it seems that, despite the difference in the relative heights of the peaks, the "redshift phenomenon" is reliably predicted by classical dynamics even at very low laser intensities. As the intensity increases, the quantum side

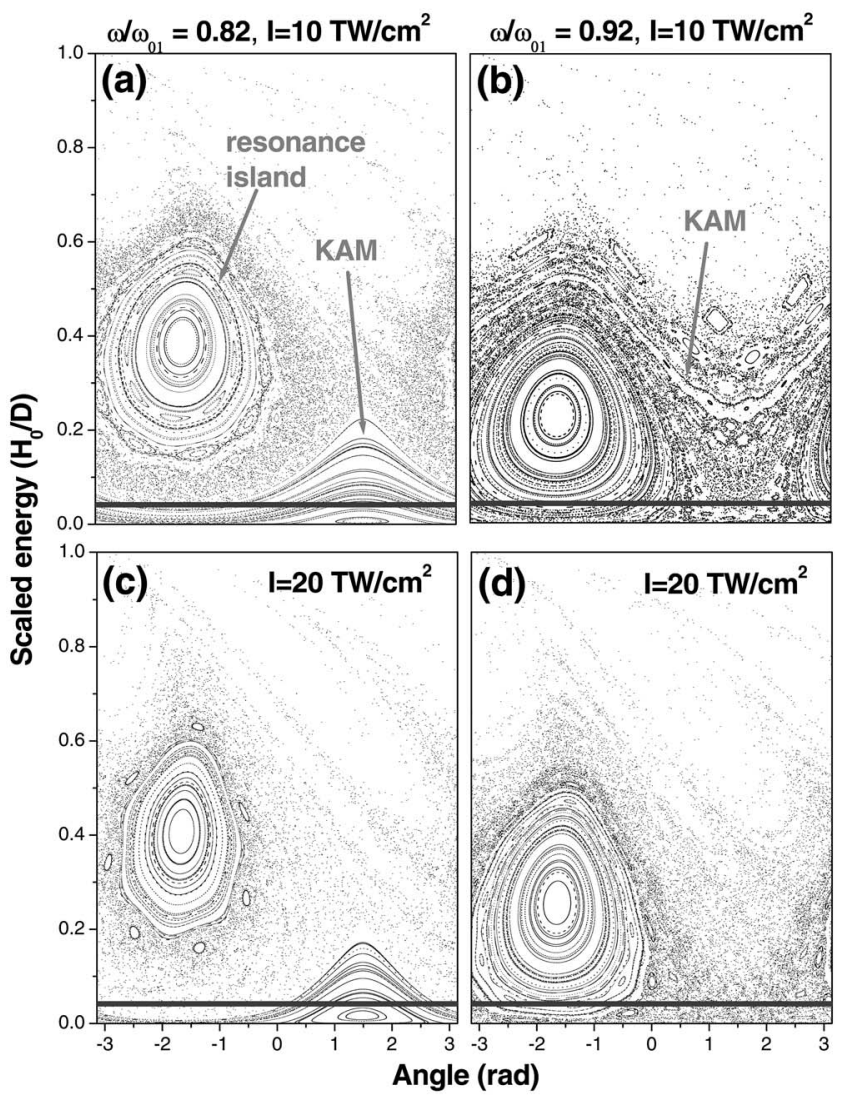

FIG. 2. Stroboscopic plots for four different laser field parameters with fixed total interaction time $t=13140$ a.u.: (a) $I$ $=10 \mathrm{TW} / \mathrm{cm}^{2}, \omega / \omega_{01}=0.82$, (b) $I=10 \mathrm{TW} / \mathrm{cm}^{2}, \omega / \omega_{01}=0.92$, (c) $I=20 \mathrm{TW} / \mathrm{cm}^{2}, \omega / \omega_{01}=0.82$, and (d) $I=20 \mathrm{TW} / \mathrm{cm}^{2}, \omega / \omega_{01}=0.92$. The gray lines depict the classical initial state.

peaks become more enhanced, while the classical peak broadens [Figs. 1(b) and 1(c)].

In what follows, we offer interpretations of the behavior of the dissociation probability versus laser frequency, in the context of both CM and QM.

A phase space analysis of the $P_{D}^{c}\left(\omega / \omega_{01}\right)$ curve for constant field intensity was given in detail in [8]. It was attributed to a transition from trapping of initial-state trajectories in Kolmogorov-Arnold-Moser (KAM) tori at low frequencies to trapping inside the island of the main resonance between the field and the internal vibrational motion at high frequencies. Between these limits, one can find intermediate frequencies at which the KAM tori have shrunk and the resonance island has not yet overlapped with the initial state. At these frequencies, the photodissociation probability is maximized. Figure 2 shows the transition from KAM to resonance trapping of the initial state (gray lines) as frequency increases, for two different intensities $\left(10\right.$ and $\left.20 \mathrm{TW} / \mathrm{cm}^{2}\right)$. The increase of intensity, while keeping the frequency constant, leads to shrinking of both KAM tori and resonance regions in phase space. This has as a consequence the enlargement of the region of intermediate frequencies with high photodissociation probability, which leads to the broadening of the $P_{D}^{c}\left(\omega / \omega_{01}\right)$ curve with laser intensity, as seen in Fig. 1 . For high frequencies, an additional stabilization mechanism 


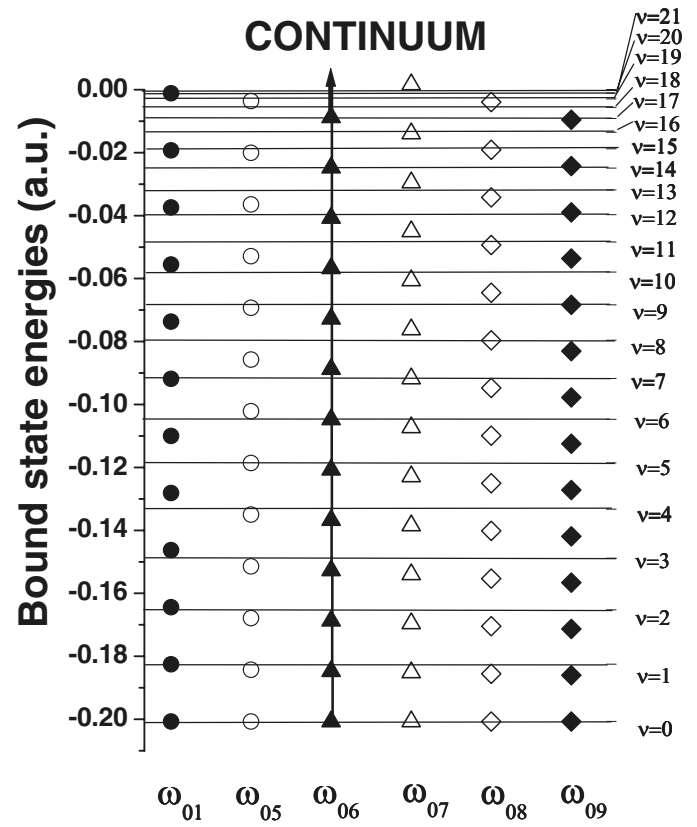

FIG. 3. Bound-state vibrational energies of the ground state of the HF molecule and resonance multiphoton ladders.

is observed. As we can see in Fig. 2(b), at low intensities, a part of the initial state, though embedded in a narrow chaotic region, cannot dissociate since it is bounded in the phase space by a remaining set of KAM tori at higher energies immersed in complex structures of high-order resonance islands. As the intensity increases, these tori start to break, forming cantori which act as partial barriers of the transport to higher energies and cause the sticking of trajectories around the resonance islands [29,30]. At even higher laser intensities [Fig. 2(d)], these barriers disappear and the chaotic trajectories diffuse freely at high energies and dissociate. The asymmetry in the $P_{D}^{c}\left(\omega / \omega_{01}\right)$ curves, i.e., the long tail at high frequencies and intensities, has been associated with the existence of these sticking phenomena [8], whose impact on dissociation dynamics will be discussed in more detail in Sec. III C.

Quantum-mechanically, resonant multiphoton absorption leads to a considerable augmentation of the photodissociation probability at certain frequencies. Indeed, the maximum which appears at $\omega / \omega_{01}=0.88$ and which is very pronounced at small intensities, as well as the side peaks, can be explained by this process, as shown in the schematic picture of Fig. 3, which depicts the resonance multiphoton ladders. This maximum corresponds to a six-photon absorption between the ground and the sixth vibrational state with frequency $\omega_{06}=\left(E_{6}-E_{0}\right) / 6=0.88 \omega_{01}$. The system needs three photons to reach the $\nu=10$ state and then, with the absorption of three more photons, it reaches the $\nu=17$ state by almost resonantly stepping on the $\nu=12,14$, and 17 states. Finally, the absorption of one more photon leads to cleavage of the molecular bond. For frequencies close to the resonant frequency $\omega_{01}$, the low and intermediate bound states are strongly populated, as we will see in the last section. The photodissociation probability is therefore reduced, due to the

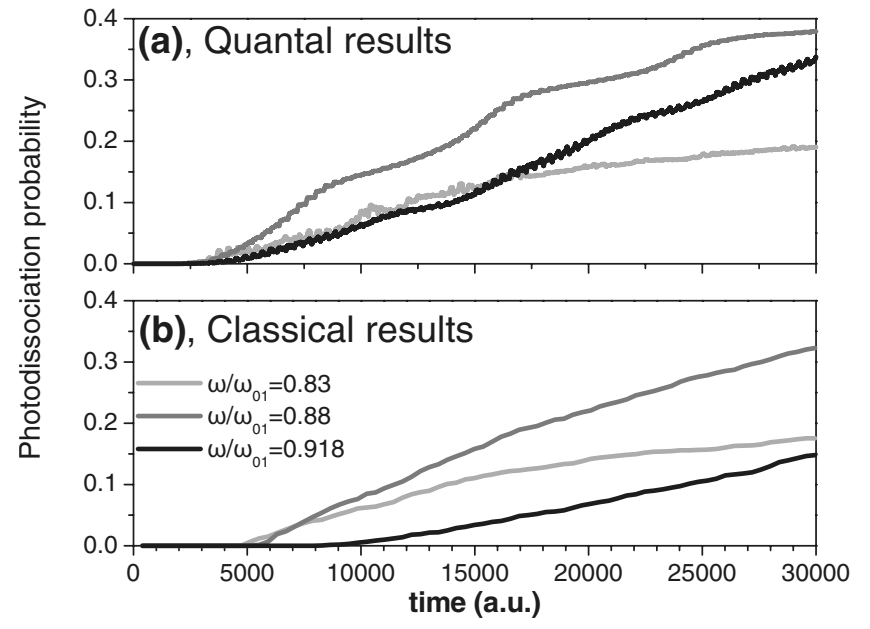

FIG. 4. Quantal (a) and classical (b) photodissociation probability for the HF molecule as a function of interaction time for three different laser frequencies $\omega / \omega_{01}=0.83$ (light gray line), 0.88 (gray line), and 0.918 (black line).

fact that the bound-state occupation probability remains trapped in these states. In addition, the photon energy does not match the energy difference of the high vibrational states, leading to a weak population of these states. It should be noted that direct transitions from the low bound states to the continuum are much less probable than those coming from higher bound states, according to their coupling matrix elements.

For frequencies lower than the $\omega_{06}$ frequency, the situation is different. As an example, we take the $\omega_{08}$ frequency for which eight photons are needed for the transition from the $\nu=0$ to the $\nu=8$ state. This is a high-order multiphoton process which becomes probable only at sufficiently high intensities (about $30 \mathrm{TW} / \mathrm{cm}^{2}$ ). Once the eighth level is reached, dissociation occurs by almost resonantly stepping on the higher bound states. This process explains the increase of the photodissociation probability around $\omega \simeq \omega_{08}\left(\omega / \omega_{01}\right.$ $\simeq 0.83$ ) which appears at high intensities. In this limit $\left(30 \mathrm{TW} / \mathrm{cm}^{2}\right)$, the form of the quantum $P_{D}\left(\omega / \omega_{01}\right)$ curve becomes more complex, since the perturbation of the energy levels is, apparently, considerable.

The results shown in Figs. 1(a)-1(c) correspond to a finite interaction time equal to 13140 a.u. In Figs. 4(a)-4(c) we show as examples the photodissociation probability versus time $P_{D}(t)$ for laser frequencies $\omega / \omega_{01}=0.83,0.88$, and 0.918 , which correspond to frequencies close to peaks of the $P_{D}^{q}$ curve in Fig. 1(b). We observe that quantal and classical $P_{D}(t)$ increase with interaction time. For $\omega / \omega_{01}=0.83$ the rate of increase of $P_{D}(t)$ decreases for long times, both quantum-mechanically and classically. This is not the case for frequencies $\omega / \omega_{01}=0.88$ and 0.918. In particular, for $\omega / \omega_{01}=0.83$ and 0.88 , the quantal and classical $P_{D}(t)$ are in good agreement, with the classical results being systematically lower than the quantal ones, while for $\omega / \omega_{01}=0.918$ the differences are larger. It should be noted that this underestimation is not a "universal" phenomenon. For example, for $\omega / \omega_{01}=0.93$ the quantal results are systematically lower than the classical ones (not shown). 


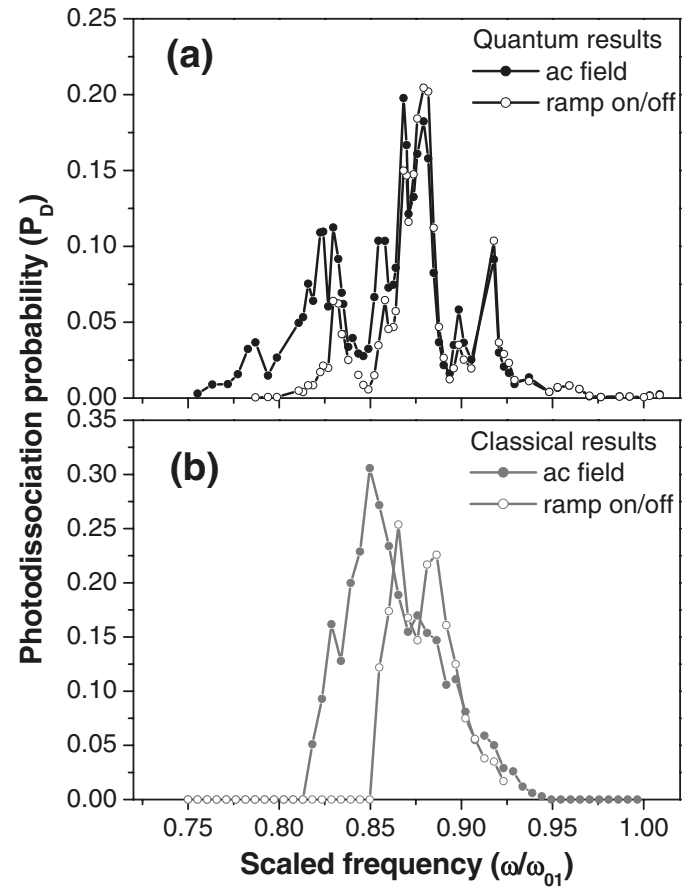

FIG. 5. Quantal (a) and classical (b) photodissociation probability for the HF molecule as a function of the scaled laser frequency. Symbols and laser intensity are the same as those used in Fig. 1(b). Open circles denote the results using a trapezoidal pulse with ramp-on time $T_{\text {on }}=4 \times 2 \pi / \omega_{01}=1570$ a.u. equal to ramp off-time $T_{o f f}$.

In conclusion, we have seen that the quantum distributions exhibit a multipeak structure, caused by the multiphoton resonant transitions, while the classical ones show a smooth curve due to the transition window between the KAM tori and the resonant island region. However, the classical model is able to qualitatively predict the gross features of the corresponding quantum distribution $P_{D}^{q}\left(\omega / \omega_{01}\right)$, i.e., the photodissociation occurs predominantly in a domain of laser frequencies smaller than the $\omega_{01}$ frequency. The classical results come closer to the quantum ones as the intensity increases.

\section{B. Dependence on the shape of the laser pulse}

In the previous section the numerical investigations were limited to the consideration of ac laser fields. However, this consideration is inadequate for short laser pulses and the effects of ramp-on and -off times have to be taken into account. In the present section, we investigate the effect of the temporal pulse shape on the photodissociation process. To this purpose, we performed calculations using a trapezoidal pulse with ramp-on and ramp-off time equal to $4 \times 2 \pi / \omega_{01}$. In Fig. 5, we compare the quantum [Fig. 5(a)] and classical [Fig. 5(b)] results of the photodissociation probability as a function of the scaled laser frequency $\omega / \omega_{01}$ for the cases of an ac and a trapezoidal pulse with intensity $I=20 \mathrm{TW} / \mathrm{cm}^{2}$. The effect of the pulse shape is apparent in both models. The quantal and classical distributions show a similar behavior:

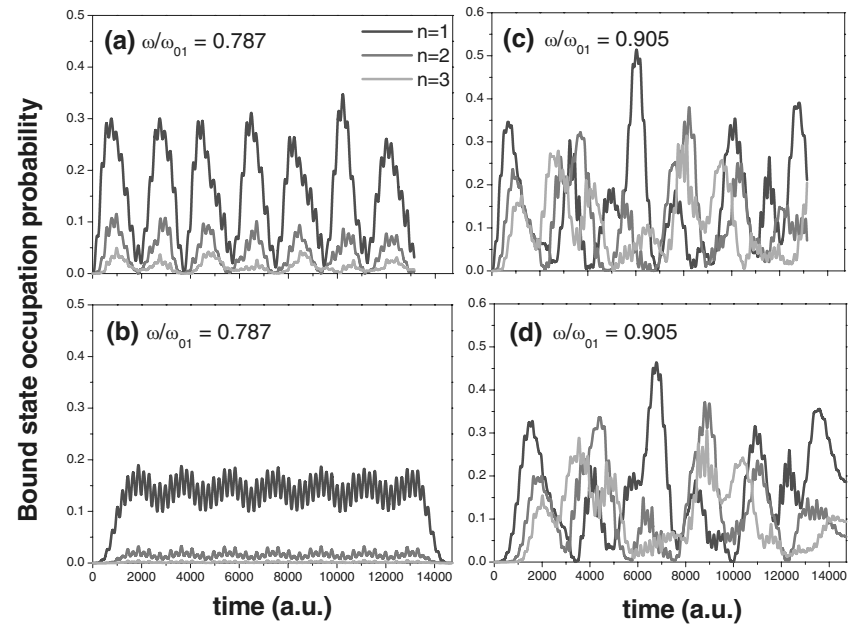

FIG. 6. Quantum bound-state occupation probabilities as a function of interaction time, for laser frequencies $\omega / \omega_{01}=0.787$ and 0.905. Dark gray line, $\nu=1$; gray line, $\nu=2$; and light gray line, $\nu$ $=3$ state. (a) and (c) correspond to an ac field and (b) and (d) correspond to a trapezoidal pulse like the one used in Fig. 5. The laser intensity is $I=20 \mathrm{TW} / \mathrm{cm}^{2}$.

at low frequencies the photodissociation probability is strongly affected by the shape of the pulse, while at higher frequencies the ramping plays a minor role.

This effect can be explained quantum-mechanically by looking at the bound-state occupation probabilities as a function of time. Figure 6 shows the results for the vibrational states $\nu=1,2,3$ as a function of time for laser frequencies $\omega / \omega_{01}=0.787$ and 0.905 . For the smaller frequency [Figs. 6(a) and 6(b)], we observe that the excited bound states are weakly populated during the interaction when a ramp-on is applied (higher bound states are not included in the figure, as they are too small to be distinguished). This is in agreement with the results of Goggin and Milonni [19], who observed that the use of the ramp-on reduces the excitation energy compared to the case of a sudden turn-on. However, this is not a general statement and the picture is different at high frequencies, close to $\omega_{01}$. The gradual turn-on of the field has a minor effect: In Figs. 6(c) and 6(d) the bound-state occupation probabilities for a trapezoidal pulse follow the corresponding ones for the ac field, delayed only by the ramp-on time.

As stated above, the asymmetry in the ramp-on time effects on $P_{D}\left(\omega / \omega_{01}\right)$ is not an exclusively quantum phenomenon, since classical dynamics also predicts it [Fig. 5(b)]. In Fig. 7, the stroboscopic plots of the phase space for $I$ $=20 \mathrm{TW} / \mathrm{cm}^{2}$ and three different scaled laser frequencies $\omega / \omega_{01}=0.84,0.89,0.92$ are included. Also, we have added the straight line of the initial state (gray line) as well as its snapshot at the end of the ramp-on time (light gray line) just before the period of constant amplitude field starts. The overlap of the latter line with the stability regions (KAM or resonant) gives the survival probability of the molecule after the end of the laser pulse. At low frequencies $(\sim 0.84)$, we see in Fig. 7(a) that, although a part of the gray line representing the initial classical state overlaps the chaotic region and thus can dissociate, the evolution of this line during ramp-on time 


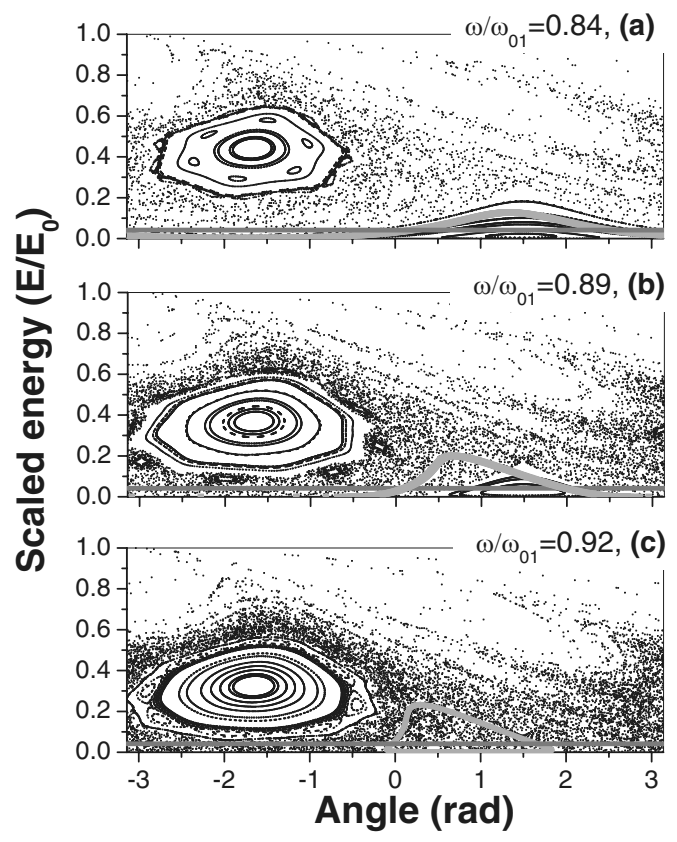

FIG. 7. Stroboscopic plots for three different ac laser field frequencies $\omega / \omega_{01}(\mathrm{a})=0.84$, (b) 0.89 , and (c) 0.92 , with laser intensity $I=20 \mathrm{TW} / \mathrm{cm}^{2}$. Gray lines and light gray lines depict the initial classical state and the classical state at the end of the ramp-on time, respectively (see text for details).

leads to a classical state (light gray line) totally trapped inside the KAM region, thereby reducing the photodissociation probability to zero. As frequency increases, two effects occur [see Fig. 7(b)]. The first is the shrinking of the KAM tori and the second is the energy broadening of the classical state after ramp-on time. The combination of these effects leads to a partial overlap of the classical state (light gray line) with the KAM stability region similar to that occurring when no ramp-on time is considered (gray line). This explains the minor effect of the shape of the laser pulse on $P_{D}^{c}\left(\omega / \omega_{01}\right)$ at high frequencies. The sticking regions in phase space present at higher frequencies [Fig. 7(c)], have even less influence on the dissociation effects of ramp-on times.

\section{Dependence of the photodissociation dynamics on duration of interaction}

\section{Survival probability versus time}

As it was pointed out by Constantoudis and Nicolaides [8], classical phase space changes can strongly influence the survival probability and also the photodissociation rate. As a matter of fact, at high frequencies, the stickiness of chaotic trajectories in the regions around the resonance islands was found to be more important than that around the KAM tori. One question that was addressed in [8] is whether there exists a quantum manifestation of the classical stickiness effects associated with the resonance islands. As these effects are expected to be pronounced at high frequencies, we performed quantal and classical calculations of the survival probability as a function of interaction time for laser frequencies near and far from the resonant $\omega_{01}$ frequency (Fig. 8).

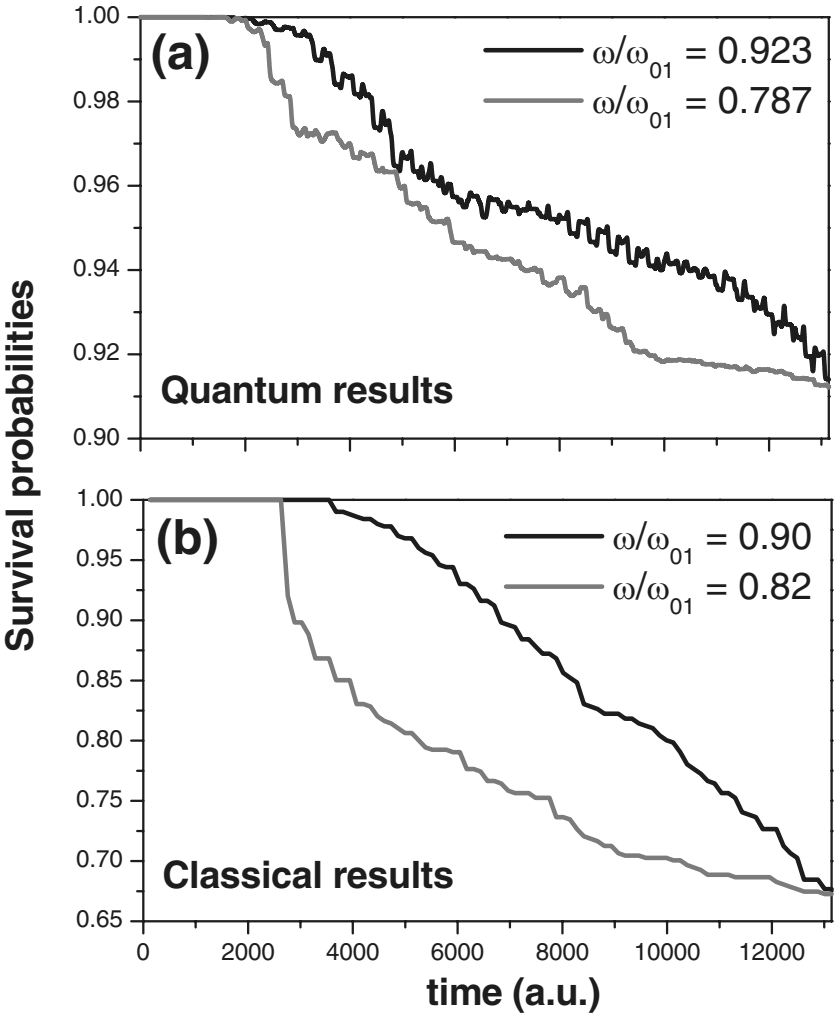

FIG. 8. Quantal (a) and classical (b) survival probabilities as a function of interaction time. Quantal results correspond to laser frequencies $\omega / \omega_{01}=0.787$ (gray line) and 0.923 (black line), while classical results correspond to laser frequencies $\omega / \omega_{01}=0.82$ (gray line) and 0.90 (black line). The laser intensity is $I=30 \mathrm{TW} / \mathrm{cm}^{2}$.

The quantum survival probability [Fig. 8(a)] for the higher frequency considered here, $\omega / \omega_{01}=0.923$, decreases slowly than the one for the lower frequency $\omega / \omega_{01}=0.787$. We have chosen these frequencies as they produce the same value of photodissociation probability at the end of the pulse. Looking at the classical results [Fig. 8(b)], the same behavior is observed, and it is even more pronounced. The explanation is based on the phase space topology: for the higher frequency, chaotic trajectories that are stuck near the resonance island and overlap with the classical initial state [see Fig. 2(d)], will dissociate at later times, leading to a small dissociation rate. On the other hand, for smaller frequencies, dissociation will occur faster due to the lack of such trajectories near the KAM tori [see Fig. 2(c)]. We therefore conclude that classical dynamics qualitatively predicts the basic features of the quantum dynamics of multiphoton dissociation.

\section{Bound-state occupation probabilities versus time}

Apart from the stickiness effects, the classical resonance region differs from KAM tori in the energy variation of trajectories versus time, i.e. the energy of resonance tori varies much more than the energy of KAM tori [Figs. 2(a)-2(d)]. In this section we analyze the consequences of this difference on the classical bound-state occupation probabilities versus time for high and low frequencies, and we investigate their possible connection with the quantum dynamics. Figure 9 shows the CM [Figs. 9(a) and 9(b)] and QM [Figs. 9(c) and 

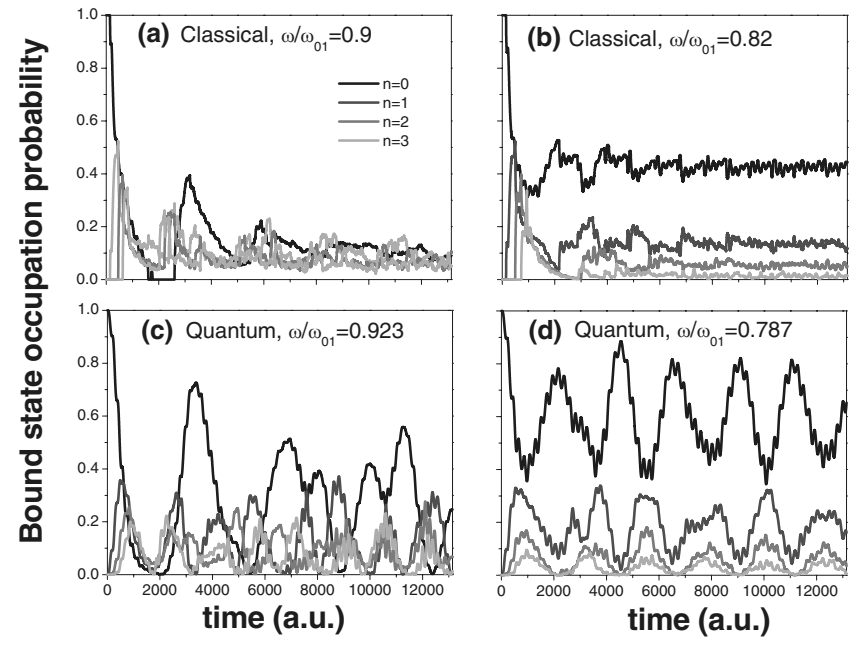

FIG. 9. Classical and quantal bound-state occupation probabilities for $\nu=0$ (black line), 1 (dark gray line), 2 (gray line), and 3 (light gray line) state, as a function of interaction time. The laser field parameters are identical to those used in Fig. 8. (a) and (b) show the classical and (c) and (d) the quantal results.

9(d)] results for the states $\nu=0,1,2,3$ for the same field parameters used in Fig. 8. In order to study the excitation probability in the classical method and to classify the trajectories corresponding to a given quantum vibrational state, we followed an approach which is well known in the area of atomic collisions [31]: A trajectory with energy $E_{c}$ corresponds to the vibrational level with energy $E_{\nu}$ when the expression $0.5\left(E_{\nu+1}+E_{\nu}\right) \geqslant E_{c} \geqslant 0.5\left(E_{\nu}+E_{\nu-1}\right)$ is satisfied. This classification holds reasonably well, especially for the low vibrational states, since they deviate only slightly from those of the harmonic oscillator. The classical explanation of the oscillations shown in Fig. 9(a) is based again on the stroboscopic plot of Fig. 2(d): at high laser frequency, the part of the initial state that will remain bound during the interaction time is trapped inside the resonance islands, which are subject to large variations in energy. However, because of their different rotating frequencies, these trajectories, will rapidly fall out of resonance, and consequently the oscillations of the classical bound-state population will be damped.

At high frequency [Fig. 9(c)], quantum-mechanically there is a strong oscillation of the ground-state probability, with an amplitude that reaches zero at certain times. At these times, population transfer to higher states takes place (not shown in the figure for $\nu>3$ for clarity reasons). Indeed, the bound-state occupation probabilities of the higher states up to $\nu=10$ also oscillate in time, showing a continuous population exchange between these states. Therefore, the major part of the quantum probability remains trapped between the low and intermediate states and only a small portion escapes to the continuum. This fact explains the small quantum dissociation probability. The oscillations observed in Fig. 9(c) are interpreted as the well-known Rabi oscillations in twostate systems [32]. Here, the system is more complicated because the laser frequency is slightly different from the $\omega_{01}$ frequency, it involves many states and also many photons, and some portion of the probability will escape to the continuum. The rather amazing finding is that such oscillations also appear in the classical calculations, and it seems that classical dynamics is able to follow the quantum evolution at least for short times of interaction [Fig. 9(a)].

At small laser frequencies, the situation is different. As shown in Fig. 9(d), the quantum-mechanical oscillations of the bound-state occupation probabilities are less pronounced and a big portion of the probability remains in the ground state, due to the mismatch of the photon energy and the energy difference between the ground and the first vibrational states. Higher vibrational states $\nu>3$ (not shown) are even less populated, and consequently the dissociation probability is small. Classical calculations reveal similar oscillations. Using as a guide the phase space of Fig. 2(c), we observe that a part of the classical phase space is trapped inside the KAM tori, which are subject to small variations in energy, and thus result in relatively smaller variations of the bound-state occupation probabilities [Fig. 9(b)], compared to the high-frequency case [Fig. 9(a)].

\section{CONCLUSIONS}

Following nonperturbative quantum and classical methods we obtained quantitative results for the multiphoton dissociation of a Morse molecule induced by ac and pulsed laser fields. Our work focused on the investigation of the quantum-classical correspondence in the region of laser frequencies $(0.8-0.9) \omega_{01}$, where dissociation is more efficient. We calculated the photodissociation probability $P_{D}\left(\omega / \omega_{01}\right)$ as a function of the laser frequency. Quantal results show a multipeak structure due to multiphoton resonance transitions, while the classical results exhibit a smooth curve, with a peak at the frequency $\omega_{\max } / \omega_{01} \simeq 0.86$ that is close to the quantum peak at $\omega_{06} / \omega_{01} \simeq 0.88$, because of the smooth transition in the phase space topology as the laser frequency is increased. Furthermore, we examined how the changes of the pulse shape affect $P_{D}\left(\omega / \omega_{01}\right)$. We found that the use of a trapezoidal pulse (instead of an ac field) influences in a similar way the form of both quantum and classical distributions $P_{D}\left(\omega / \omega_{01}\right)$, i.e., dissociation is suppressed at low frequencies while no considerable changes were found for the higher frequencies.

Finally, we investigated the survival and bound-state occupation probabilities as a function of interaction time for laser frequencies near $\omega_{\max }$. Quantal and classical survival probabilities show similar behavior: they decrease faster at low $\left(\omega<\omega_{\max }\right)$ than at high $\left(\omega>\omega_{\max }\right)$ frequencies. This finding is more pronounced in the classical calculations and the explanation is based on the slow escape of the chaotic trajectories which are located close to the resonance island. At high frequencies, we observed quantum-mechanical multiphoton Rabi oscillations where more than two levels are involved. The classical dynamics seems to be able to reproduce such oscillations due to large energy variations in the resonance island, at least for small times of interaction. At low frequencies, the oscillations of the quantal bound-state occupation probabilities are less pronounced. The classical dynamics also catches these features, which are explained by the small energy variations of the KAM tori. 


\section{ACKNOWLEDGMENTS}

This work was supported by the program "Pythagoras" which is co-funded by the European Social Fund (75\%) and National Resources (25\%).

\section{APPENDIX: MATRIX ELEMENTS FOR CONTINUUM- CONTINUUM TRANSITIONS}

We describe the calculation of the continuum-continuum matrix elements

$$
C\left(E, E^{\prime}\right)=\int_{0}^{+\infty} d x \Psi_{E}^{*}(x)\left(x-x_{0}\right) \Psi_{E^{\prime}}(x)
$$

where

$$
\begin{aligned}
\Psi_{E^{\prime}}(x)= & \sqrt{\frac{1}{2 \pi \alpha}} \sqrt{\frac{d k^{\prime}}{d E^{\prime}}}\left|A\left(\epsilon^{\prime}\right)\right|^{-1} e^{-z / 2} \\
& \times\left[A\left(\epsilon^{\prime}\right) z^{\imath \epsilon^{\prime}}{ }_{1} F_{1}\left(\frac{1}{2}-d+\imath \epsilon^{\prime}, 1+2 \imath \epsilon^{\prime}, z\right)\right. \\
& \left.+A\left(\epsilon^{\prime}\right)^{*} z^{-l \epsilon^{\prime}}{ }_{1} F_{1}\left(\frac{1}{2}-d-\imath \epsilon^{\prime}, 1-2 \imath \epsilon^{\prime}, z\right)\right]
\end{aligned}
$$

is the free Morse molecule wave function of the continuum state [33] with energy $E^{\prime}$ and $\Psi_{E}^{*}(x)$ is the complex conjugate $\left[z=2 d e^{-\alpha\left(x-x_{0}\right)}, d=2 \mu E^{\prime} / \alpha, \epsilon^{\prime}=\sqrt{2 \mu E^{\prime} / \alpha^{2} \hbar^{2}}, A\left(\epsilon^{\prime}\right)\right.$ $=\Gamma\left(-2 \imath \epsilon^{\prime}\right) / \Gamma\left(0.5-d-\imath \epsilon^{\prime}\right), \Gamma$ is the gamma function, and ${ }_{1} F_{1}$ is the confluent hypergeometric function [28]]. According to the procedure developed for atomic targets [15], we separate the integral of Eq. (A1) into two parts: (a) the "inner part" where we numerically calculate the wave function up to a given interatomic distance $X_{c}$, above which the potential can be ignored and (b) the "outer part" which we calculate analytically for distances larger than $X_{c}$. The matrix element is written as

$$
\begin{aligned}
C\left(E, E^{\prime}\right)= & C_{\text {in }}\left(E, E^{\prime}\right)+C_{\text {out }}\left(E, E^{\prime}\right) \\
= & \int_{0}^{X_{c}} d x \Psi_{E}^{*}(x)\left(x-x_{0}\right) \Psi_{E^{\prime}}(x) \\
& +\int_{X_{c}}^{+\infty} d x \Psi_{E}^{*}(x)\left(x-x_{0}\right) \Psi_{E^{\prime}}(x) .
\end{aligned}
$$

For distances larger than $X_{c}$, the wave function is expressed as plane waves given by the expression

$$
\begin{aligned}
\lim _{x \rightarrow+\infty} \Psi_{E^{\prime}}(x)= & \sqrt{\frac{1}{2 \pi \alpha}} \sqrt{\frac{d k^{\prime}}{d E^{\prime}}}\left(e^{l\left\{\epsilon^{\prime}\left[-\alpha\left(x-x_{0}\right)+\ln (2 d)\right]+\delta_{\epsilon^{\prime}}\right\}}\right. \\
& +e^{-l\left\{\epsilon^{\prime}\left[-\alpha\left(x-x_{0}\right)+\ln (2 d)\right]+\delta_{\left.\epsilon^{\prime}\right\}}\right)}
\end{aligned}
$$

where $\delta_{\epsilon^{\prime}}=\tan ^{-1}\left\{\operatorname{Im}\left[A\left(\epsilon^{\prime}\right)\right] / \operatorname{Re}\left[A\left(\epsilon^{\prime}\right)\right]\right\}$ is the phase shift. This allows the analytic calculation of the second term in (A3).

The first term presents no difficulty, and so the wave functions and the integral are calculated numerically. However, the second term contains an on-shell singularity of the form $1 /\left(E-E^{\prime}\right)^{2}$ when $E=E^{\prime}$. In order to overcome this problem we insert a regularization factor $\exp -(\lambda x)$ in the integrand of the second term of (A1). The integral $C_{\text {out }}\left(E, E^{\prime}\right)$ $=\int_{X_{c}}^{+\infty} d r \Psi_{E}^{*}(x)\left(x-x_{0}\right) \exp -(\lambda x) \Psi_{E^{\prime}}(x)$ is calculated analytically. The justification of the value of $\lambda$ is given below. As this singularity appears inside the integral of Eq. (7), one must also think how to integrate in energy the on-shell matrix elements. The first step is to write the integral on the singularity $E=E_{n}$ as

$$
I_{\text {sing }}=\int_{E_{n-1}}^{E_{n+1}} b\left(E^{\prime}, t\right)\left[C_{\text {in }}\left(E_{n}, E^{\prime}\right)+C_{\text {out }}\left(E_{n}, E^{\prime}\right)\right] d E^{\prime} .
$$

The energy spacing was chosen small enough in order to take into account correctly the behavior of the matrix elements near the singularity, which is mathematically given by the derivative of a $\delta$ function. This behavior depends on the value of $\lambda$ in the regularization factor inside the integrand of $C_{\text {out }}\left(E_{n}, E^{\prime}\right)$. After numerical trials the value of $\lambda=10^{-3}$ was found to be adequate. For smaller values, no considerable differences were observed in $I_{\text {sing }}$. As $b\left(E^{\prime}, t\right)$ is an unknown parameter inside the integral, we approximate it by a parabolic function in energy, centered at the singular point $E_{n}$, $b\left(E^{\prime}, t\right)=A\left(E^{\prime}-E_{n}\right)^{2}+B\left(E^{\prime}-E_{n}\right)+D$, with $A, B, D$ coefficients that depend on the analytically known parameters $b\left(E_{n}, t\right), b\left(E_{n+1}, t\right), b\left(E_{n-1}, t\right)$. Then we insert this function in Eq. (A5) and calculate the three integrals. We note that the above procedure was applied only for the on-shell matrix elements. For $E \neq E^{\prime}$, the integral in energy of Eq. (7) presents no difficulty, and so it was calculated using the standard trapezoidal rule.
[1] J. E. Bayfield and P. M. Koch, Phys. Rev. Lett. 33, 258 (1974).

[2] J. G. Leopold and I. C. Percival, J. Phys. B 12, 709 (1979).

[3] G. Casati, B. V. Chirikov, D. L. Shepelyansky, and I. Guarneri, Phys. Rep. 154, 77 (1987).

[4] P. M. Koch and K. A. H. van Leeuwen, Phys. Rep. 255, 289 (1995).

[5] R. V. Jensen, S. M. Susskind, and M. M. Sanders, Phys. Rep. 201, 1 (1991).
[6] M. M. Sanders and R. V. Jensen, Am. J. Phys. 64, 21 (1996).

[7] V. Constantoudis and C. A. Nicolaides, Phys. Rev. A 55, 1325 (1997).

[8] V. Constantoudis and C. A. Nicolaides, Phys. Rev. E 64, 056211 (2001).

[9] V. Constantoudis and C. A. Nicolaides, J. Chem. Phys. 122, 84118 (2005).

[10] M. D. Feit, J. R. Fleck, and A. Steiger, J. Comput. Phys. 47, 
412 (1982).

[11] C. Leforestier and R. E. Wyatt, J. Chem. Phys. 78, 2334 (1983).

[12] R. Heather and H. Metiu, J. Chem. Phys. 88, 5496 (1988).

[13] A. Guldberg and G. D. Billing, Chem. Phys. Lett. 186, 229 (1991).

[14] K. Sugimori, T. Ito, Y. Takata, K. Ichitani, H. Nagao, and K. Nishikawa, Int. J. Quantum Chem. 106, 3079 (2006).

[15] Th. Mercouris, Y. Komninos, S. Dionissopoulou, and C. A. Nicolaides, Phys. Rev. A 50, 4109 (1994).

[16] C. A. Nicolaides, Th. Mercouris, and I. Petsalakis, Chem. Phys. Lett. 212, 685 (1993).

[17] E. F. Lima and J. E. M. Hornos, J. Chem. Phys. 125, 164110 (2006).

[18] P. C. Dardi and S. K. Gray, J. Chem. Phys. 77, 1345 (1982).

[19] M. E. Goggin and P. W. Milonni, Phys. Rev. A 37, 796 (1988); 38, 5174 (1988).

[20] P. M. Morse, Phys. Rev. 34, 57 (1929).

[21] J. R. Stine and D. W. Noid, Opt. Commun. 31, 161 (1979).
[22] J. J. Tanner and M. M. Maricq, Phys. Rev. A 40, 4054 (1989).

[23] R. Graham and M. Hohnerbach, Phys. Rev. A 45, 5078 (1992).

[24] J. A. C. Gallas, Phys. Rev. A 21, 1829 (1980).

[25] A. Matsumoto, J. Phys. B 21, 2863 (1988).

[26] K. Iwamoto and A. Matsumoto, J. Phys. B 30, L335 (1997).

[27] E. F. Lima and J. E. M. Hornos, J. Phys. B 38, 815 (2005).

[28] Handbook of Mathematical Functions, edited by M. Abramowitz and I. A. Stegun (Dover, New York, 1972).

[29] C. Efthymiopoulos, G. Contopoulos, N. Voglis, and R. Dvorak, J. Phys. A 30, 8167 (1997).

[30] G. Contopoulos, M. Harsoula, N. Voglis, and R. Dvorak, J. Phys. A 32, 5213 (1999).

[31] B. H. Bransden and M. R. C. McDowel, Charge Exchange and Theory of Ion-Atom Collisions (Clarendon Press, Oxford, 1992).

[32] I. I. Rabi, Phys. Rev. 51, 652 (1937).

[33] R. W. Nicholls, Chem. Phys. Lett. 79, 317 (1981). 